\title{
Prolonged Survival in Patients with Metastatic HER2-Positive Inflammatory Breast Cancer: A Case Report and Review of the Literature
}

\author{
Jennifer Y. Ge $e^{a, b}$, c Beth Overmoyer ${ }^{a, b}$ \\ aSusan F. Smith Center for Women's Cancers, Dana-Farber Cancer Institute, Boston, MA, \\ USA; ${ }^{b}$ Department of Medical Oncology, Dana-Farber Cancer Institute, Boston, MA, USA; \\ 'Harvard-MIT Division of Health Sciences and Technology, Harvard Medical School, Boston, \\ MA, USA
}

\section{Keywords}

Inflammatory breast cancer - HER2-positive breast cancer - Anti-HER2 therapy - Metastatic disease · Stage IV

\begin{abstract}
Inflammatory breast cancer (IBC) is a rare type of breast cancer that is associated with poor outcomes compared with non-IBC. Overexpression of HER2 is enriched in IBC, and those with HER2-positive disease have a relatively favorable prognosis, with improved survival over the last two decades driven by the advent of novel targeted therapies. Here, we present two patients who have survived for over 10 years after being diagnosed with de novo metastatic HER2-positive IBC. We review the data for the treatments available for metastatic HER2-positive IBC and the evolving treatment recommendations for this disease.
\end{abstract}

\section{Introduction}

Inflammatory breast cancer (IBC) is a rare type of breast cancer that comprises $2-4 \%$ of all breast cancer cases in the USA [1]. It is diagnosed by its clinical presentation of erythema, edema, and peau d'orange involving more than one-third of the breast, as well as a rapid onset of symptoms (<6 months) [1]. Imaging findings of skin thickening and abnormal parenchymal enhancement may support the diagnosis, and pathologically, skin biopsies may show invasion of tumor emboli into the dermal-lymphatic system [1]. IBC tends to affect younger women 
and has worse clinical outcomes compared with non-IBC, with a median survival of 4.8 years for stage III and 2.3 years for stage IV disease [1]. In addition, among patients diagnosed with IBC, there is a higher incidence of de novo metastatic disease compared with those with non-IBC (about 20\% vs. 4\%) [2]. Thus, IBC leads to a disproportionate number of breast cancer-related deaths despite its low incidence.

Like all breast cancers, IBC is classified according to its estrogen receptor (ER), progesterone receptor (PR), and human epidermal growth factor receptor 2 (HER2) status. Amongst IBC tumors, there is a high prevalence of HER2 positivity, approximately $35 \%$ compared to $20 \%$ in non-IBC [2-4]. Interestingly, the HER2-positive subtype is associated with a more favorable prognosis compared with other subtypes of IBC [2, 3]. Among patients with stage IV IBC, those with HER2-positive disease have a 5-year overall survival (OS) ranging from $30 \%$ to $45 \%$ depending upon the hormone receptor (HR), i.e., ER and PR status, compared with $35 \%$ and $15 \%$ in HR-positive, HER2-negative and triple-negative (ER/PR and HER2negative) IBC, respectively [4]. Furthermore, unlike in non-IBC, HR positivity does not seem to confer an additional survival benefit amongst those with HER2-positive IBC $[3,4]$.

These relatively favorable outcomes in HER2-positive metastatic IBC are attributed to the success of targeting HER2 as a therapeutic approach, as well as the number of new antiHER2-targeting agents that have become available over the past decade. Here, we present two patients with HER2-positive IBC with visceral metastases present at the time of diagnosis who have survived for over 10 years. While both have had a remarkably long course, one case demonstrates the cumulative benefit that is possible with our growing number of treatment options, while the second illustrates the extent of the response that patients can have from a single agent.

\section{Case Reports}

\section{Patient 1}

A 61-year-old woman presented in 2007 with 2-3 weeks of left breast swelling and tenderness and on exam was found to have an enlarged left breast with thickened skin, mild erythema, and edema throughout, as well as palpable left axillary lymph nodes. A core biopsy of the left breast showed invasive ductal carcinoma which was ER-low positive (2\%), PR-negative, and HER2-positive (IHC 3+; FISH ratio, 7.1). On staging studies, she was found to have enlarged ipsilateral axillary and supraclavicular lymph nodes, as well as a $1.3-\mathrm{cm}$ mass in liver segment V (Fig. 1a). This was biopsied and revealed carcinoma staining positive for HER2 and negative for ER, consistent with metastatic HER2-positive IBC. Her initial treatment consisted of 12 weeks of paclitaxel and trastuzumab, with a partial response in all lesions, followed by maintenance trastuzumab. Restaging imaging performed after 1 year of trastuzumab monotherapy demonstrated a complete response in her liver metastasis (Fig. 1b).

After 3.5 years of single-agent trastuzumab and continued complete clinical response of her systemic disease, the decision was made to pursue locoregional treatment with a modified radical mastectomy. The mastectomy contained a $0.5-\mathrm{cm}$ focus of residual invasive ductal carcinoma which was HER2-positive and HR-negative and 0 of 13 left axillary lymph nodes involved with disease. Following surgery, she continued on maintenance trastuzumab.

After 2 additional years on single-agent trastuzumab, the patient's disease metastasized to the lungs, which was confirmed by video-assisted thorascopic surgery to be HER2-positive (FISH ratio, 4.0) metastatic IBC. Her clinical course over the next 4 years consisted of six additional lines of combination chemotherapy with anti-HER2 therapy, including the anti-HER2 antibody drug conjugate (ADC) trastuzumab emtansine (T-DM1). The patient eventually

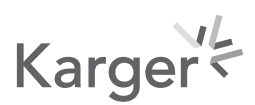




\section{Case Reports in Oncology}

\begin{tabular}{l|l}
\hline Case Rep Oncol 2021;14:1071-1079 \\
\hline DOI: 10.1159/000516760 & $\begin{array}{l}\odot \text { 2021 The Author(s). Published by S. Karger AG, Basel } \\
\text { www.karger.com/cro }\end{array}$ \\
\hline
\end{tabular}

Ge and Overmoyer: Prolonged Survival in Metastatic HER2+ Inflammatory Breast Cancer
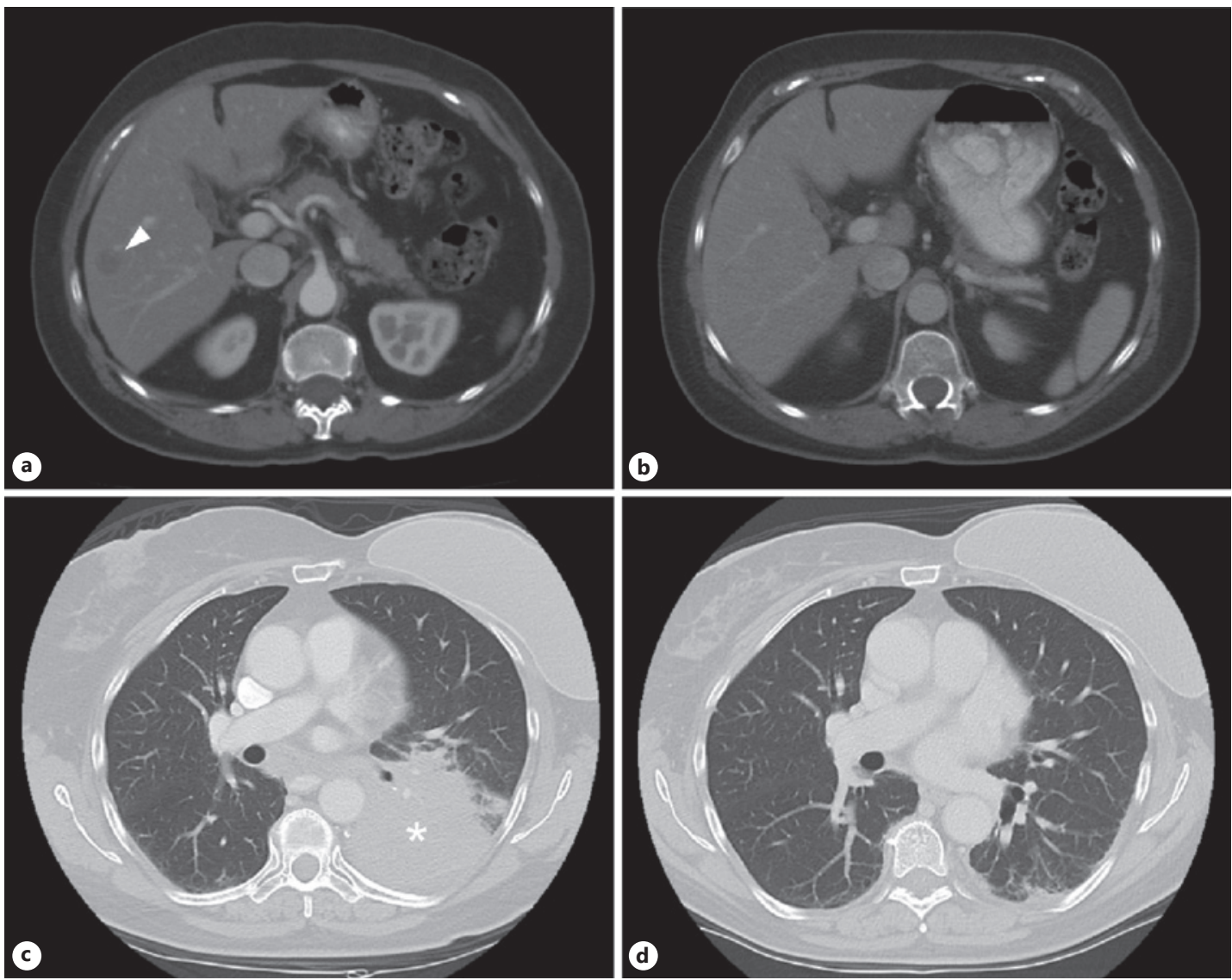

Fig. 1. Complete response of metastatic disease in patient 1. a A 1.3-cm mass in the liver at the time of diagnosis (arrowhead). b Complete resolution of the lesion after neoadjuvant therapy with 12 weeks of paclitaxel and trastuzumab, followed by 1 year of trastuzumab monotherapy. c Left lower lobe mass $\left(^{*}\right)$ that progressed on multiple lines of therapy, prior to initiating trastuzumab deruxtecan. $\mathbf{d}$ Complete response in the lung after 1 year of trastuzumab deruxtecan.

enrolled in a phase II clinical trial of trastuzumab deruxtecan (DS-8201, NCT03248492, Daiichi Sankyo/AstraZeneca), resulting in a complete response in her pulmonary lesions (Fig. 1c, d). Unfortunately, after responding for more than 1.5 years on this therapy, the patient succumbed to drug-induced pneumonitis, rather than metastatic IBC. Her OS from date of diagnosis was 12 years and 3 months.

\section{Patient 2}

A 42-year-old woman presented in 2010 with 2-3 days of worsening edema, tenderness, and erythema of her left breast. On exam, she had an enlarged left breast with a peau d'orange appearance, mild erythema over the lower half, nipple retraction, and palpable left axillary lymph nodes. A core needle biopsy of the left breast and fine needle aspiration of an axillary lymph node revealed invasive ductal carcinoma which was HR-negative and HER2-positive (FISH ratio, 8.3). Staging scans demonstrated numerous subcentimeter pulmonary nodules (Fig. 2a), which were confirmed to be HER2-positive (IHC 3+) metastatic breast cancer via a video-assisted thorascopic surgery procedure.

The patient initiated treatment on paclitaxel and trastuzumab and then switched to docetaxel, carboplatin, and trastuzumab. Following 6 cycles ( 18 weeks) of treatment, restaging studies demonstrated a complete response of her systemic disease (Fig. 2b) but persistent 

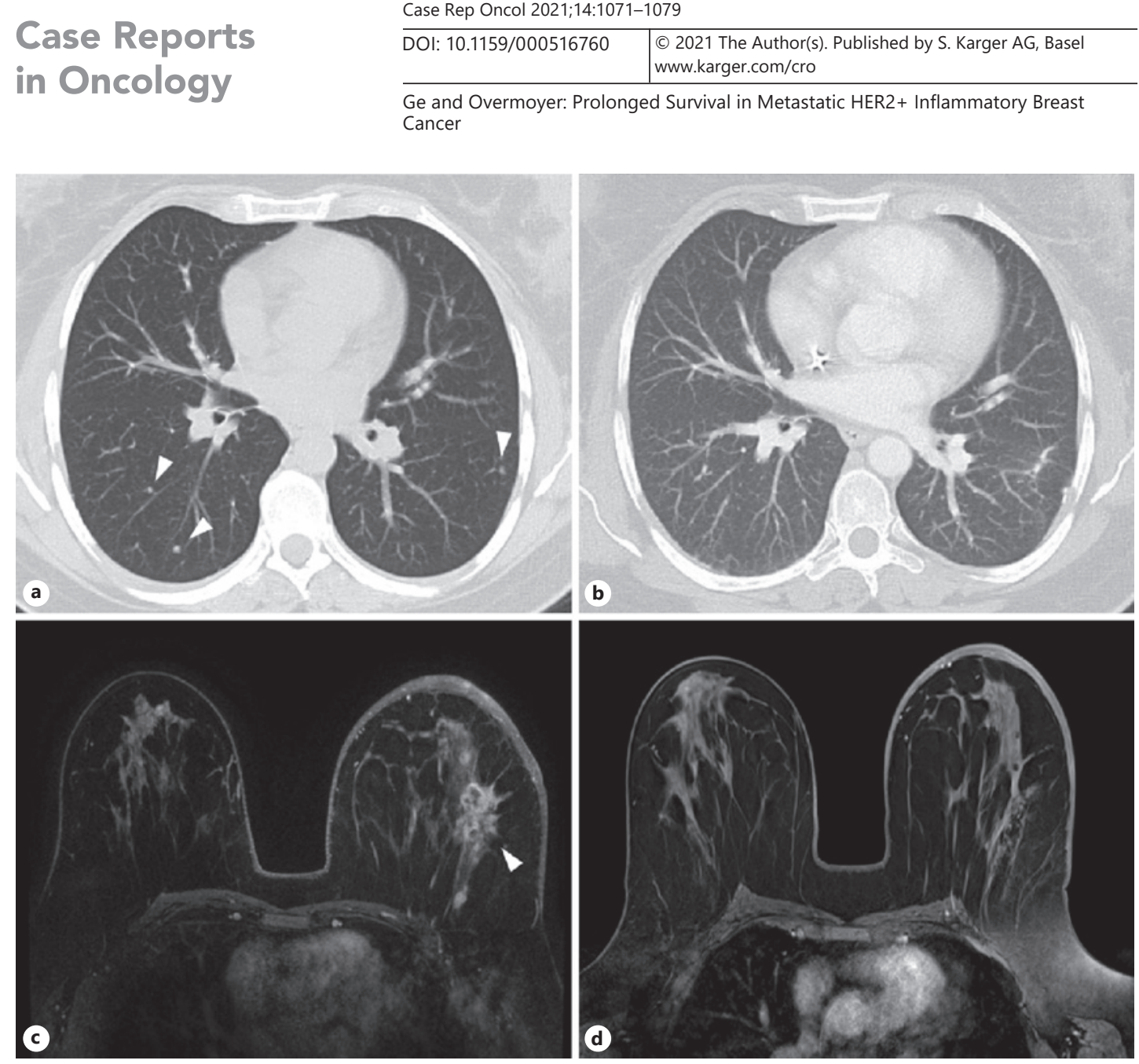

Fig. 2. Response to neoadjuvant systemic therapy in patient 2. a Multiple subcentimeter pulmonary nodules at the time of diagnosis (arrowheads). b Complete response of the lung disease after neoadjuvant therapy with 6 cycles of docetaxel, carboplatin, and trastuzumab. c Initial breast MRI with a larger left breast with an abnormal enhancing mass (arrowhead) and diffuse skin thickening. $\mathbf{d}$ Resolution of the mass after neoadjuvant chemotherapy but persistent skin thickening.

skin thickening of the left breast (Fig. 2c, d). Because of the concern for residual disease, the patient underwent locoregional therapy with radiation to the breast (5,000 cGy) and regional lymph nodes $(4,600 \mathrm{cGy})$, while continuing to receive trastuzumab monotherapy. After completing radiation and 2 months of maintenance trastuzumab, she had sufficient local disease response to proceed to a left modified radical mastectomy. The pathology showed a pathologic complete response, i.e., no residual invasive carcinoma in the resected breast or in 11 axillary lymph nodes. At the time of this publication, she remains on trastuzumab with no evidence of disease, 10 years and 10 months out from her initial diagnosis.

\section{Discussion}

The treatment of HER2-positive breast cancer has changed dramatically in the last 20 years with the advent of anti-HER2-targeted therapies. Trastuzumab, the first humanized monoclonal antibody against the extracellular domain of HER2, was FDA-approved in the USA in 1998, following the pivotal trial demonstrating an OS advantage among those receiving combination chemotherapy with trastuzumab [5]. Trastuzumab's marked efficacy in HER2positive IBC was demonstrated in the phase III NOAH trial, which included the largest number 
of IBC patients in any HER2-directed therapy trial [6]. This study found that the addition of trastuzumab to neoadjuvant chemotherapy in newly diagnosed patients increased the OS from $44 \%$ to $74 \%$ at 5 years in the inflammatory subgroup [6]. Thus, trastuzumab is recommended for all patients diagnosed with HER2-positive IBC, though notably the NOAH trial did not include any patients with metastatic disease.

A second humanized monoclonal antibody, pertuzumab (Genentech), was later developed, which also binds to the extracellular domain of HER2 but to a different domain than trastuzumab. Pertuzumab was approved in 2012 for metastatic HER2-positive breast cancer, based on the results of the CLEOPATRA trial [7]. This study showed that the addition of pertuzumab to trastuzumab and docetaxel increased the median OS of patients with metastatic breast cancer from 40.8 to 56.5 months (hazard ratio [HR], 0.68; 95\% confidence interval [CI]: $0.56-$ $0.84 ; p<0.001$ ) [7]. Although this study did not specifically evaluate participants with IBC, this regimen is nevertheless recommended as the first-line therapy for metastatic HER2positive IBC.

More recently, monoclonal antibodies linked to a chemotherapy payload, known as ADCs, have gained much attention for their potency and efficacy, particularly in the treatment of HER2-positive breast cancer. Trastuzumab emtansine (Genentech), also known as T-DM1, was FDA-approved in 2013 as the first drug of this class for HER2-positive metastatic breast cancer. In the phase III EMILIA trial, trastuzumab emtansine was shown to increase OS in patients with advanced HER2-positive disease previously treated with trastuzumab and a taxane from 25.1 to 30.9 months (HR, 0.68 ; 95\% CI: 0.55-0.85; $p<0.001$ ) compared to lapatinib and capecitabine [8]. Furthermore, the phase III TH3RESA trial found that trastuzumab emtansine also prolonged OS in patients previously treated with trastuzumab, lapatinib, and a taxane to 22.7 months, compared to 15.8 months with physician's choice of treatment (HR, 0.68 ; $95 \%$ CI: $0.54-0.85 ; p=0.0007$ ] [9]. Thus, trastuzumab emtansine is now often preferred as a second-line therapy for metastatic HER2-positive breast cancer.

In late 2019, a second ADC, trastuzumab deruxtecan (Daiichi Sankyo/AstraZeneca), was granted accelerated approval for the treatment of metastatic HER2-positive breast cancer that has progressed after receiving other anti-HER2 therapies. This was based on the results of the phase II DESTINY-Breast01 trial, which demonstrated a $60.9 \%$ overall response rate (ORR) in patients previously treated with trastuzumab emtansine [10]. Notably, response rates were equivalent in those who had received trastuzumab emtansine immediately prior to receiving trastuzumab deruxtecan, despite the fact that the two drugs use identical antibodies [10]. The comparable efficacy in these patients is attributed to the drug's novel and more potent chemotherapeutic agent, deruxtecan, as well as the increased payload to antibody ratio and release of the drug to neighboring cells, i.e., the bystander effect [10].

Another method of targeting HER2 is through the use of oral small molecule inhibitors. Lapatinib (GlaxoSmithKline), a tyrosine kinase inhibitor that inhibits both HER2 and EGFR, was FDA-approved in 2007 in combination with capecitabine as a second-line therapy for metastatic HER2-positive breast cancer, after a phase III trial demonstrated a 4-month increase in progression-free survival (PFS) compared with capecitabine alone [11]. Furthermore, a phase II IBC-specific study found that lapatinib monotherapy had a 39\% ORR and led to a 14.6-week median PFS in patients previously treated with anthracycline, taxane, and trastuzumab, though this trial included both those with stage III and IV disease [12]. However, neratinib (Puma Biotechnology), an irreversible pan-HER tyrosine kinase inhibitor, has since been shown to have superior efficacy compared with lapatinib. In the phase III NALA trial, neratinib significantly improved mean PFS compared to lapatinib, from 6.6 to 8.8 months (HR, 0.76; 95\% CI: 0.63-0.93; $p=0.0059$ ), when combined with capecitabine among patients with metastatic breast cancer previously treated with at least 2 anti-HER2 therapies [13]. More recently, tucatinib (Seagen) was FDA-approved in 2020 in combination with capecitabine and

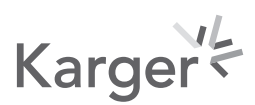


trastuzumab as another option for later-line anti-HER2 therapy, based on the results of the phase II HER2CLIMB trial [14]. This study showed improved median OS from 17.4 to 21.9 months (HR, 0.66; 95\% CI: 0.50-0.88; $p=0.005$ ) among patients previously treated with trastuzumab, pertuzumab, and trastuzumab emtansine [14]. Tucatinib is also highly selective for HER2 with minimal inhibition of EGFR, compared with lapatinib and neratinib, thus improving its toxicity profile [14].

The newest systemic treatment for metastatic HER2-positive breast cancer, margetuximab (MacroGenics), was approved in December 2020. Margetuximab is a chimeric Fc-engineered immune-activating antibody against HER2 that targets the same epitope as trastuzumab. Its approval was based on the results of the phase III SOPHIA trial, which showed an improvement in median PFS from 4.9 to 5.8 months (HR, 0.76 ; 95\% CI: 0.59-0.98; $p=0.03$ ) in patients with advanced HER2-positive breast cancer treated with at least 2 prior anti-HER2 therapies [15]. Although the benefit of margetuximab appears to be minimal, the rationale behind its design is interesting, as its Fc region was engineered to increase its affinity for activating over inhibitory $\mathrm{Fc} \gamma$ receptors, in order to modulate the immune response [15]. Such immunomodulatory agents may prove to have a unique role in IBC, which has been found to have fewer activated T cells and antigen-presenting cells, along with increased levels of PD-L1 expression and numbers of immunosuppressive tumor-associated macrophages, compared with non-IBC [1].

While these expanding treatment options (Table 1) have led to exceptional improvements in outcomes, the major limitation of our current approach to metastatic HER2-positive IBC is that treatment recommendations are based on studies with predominantly non-IBC cohorts. Due to the rarity of IBC and thus the difficulty in recruiting these patients, there is limited evidence to validate the efficacy of these anti-HER2 therapies in IBC. NOAH was one of the few pivotal trials to recruit enough IBC patients for a subgroup analysis [6], but still, this study did not include those with metastatic disease. More trials specifically for IBC will be needed, in order to improve our understanding of the differences between IBC and non-IBC and to identify optimal treatment strategies for IBC.

Several questions remain to be answered regarding the optimal treatment strategy for HER2-positive IBC in the metastatic setting. Although it has been shown that patients with stage III IBC have improved OS with trimodal therapy, i.e., preoperative chemotherapy, surgery, and radiation, the benefit of locoregional therapy with surgery and radiation for stage IV IBC remains uncertain [4]. As illustrated by our two cases, patients with metastatic IBC can achieve an excellent response with initial systemic therapy, followed by a long period without disease progression or with stable disease on trastuzumab alone. It remains to be seen whether those patients experiencing a prolonged remission can be considered cured and whether there is a subset in whom maintenance trastuzumab, with or without pertuzumab, can be discontinued. Further study will also be needed to determine the optimal sequence of the newer regimens, as well as the integration of chemotherapy with HER2targeting agents.

While the efficacy of systemic anti-HER2 therapies is improving, achieving adequate control of central nervous system (CNS) metastases still poses a significant challenge. Among patients diagnosed with metastatic HER2-positive IBC, the rate of early CNS metastasis is very high, with cumulative incidences of $17 \%$ and $34 \%$ at 1 and 2 years, respectively [16]. Though patients with treated or stable CNS lesions are often allowed to enroll in clinical trials, the numbers are usually too small to assess the intracranial efficacy of these systemic therapies. An important exception is the HER2CLIMB trial, which included 291 patients with stable, progressing, or untreated brain metastases due to HER2-positive breast cancer [17]. The results showed that the addition of tucatinib to capecitabine and trastuzmab among these patients improved OS by 6.1 months compared with capecitabine and trastuzumab alone (HR, 0.58 ; $95 \%$ CI: $0.40-0.85 ; p=0.005$ ] [17]. Thus, systemic therapy with tucatinib, trastuzumab,

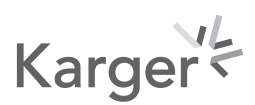




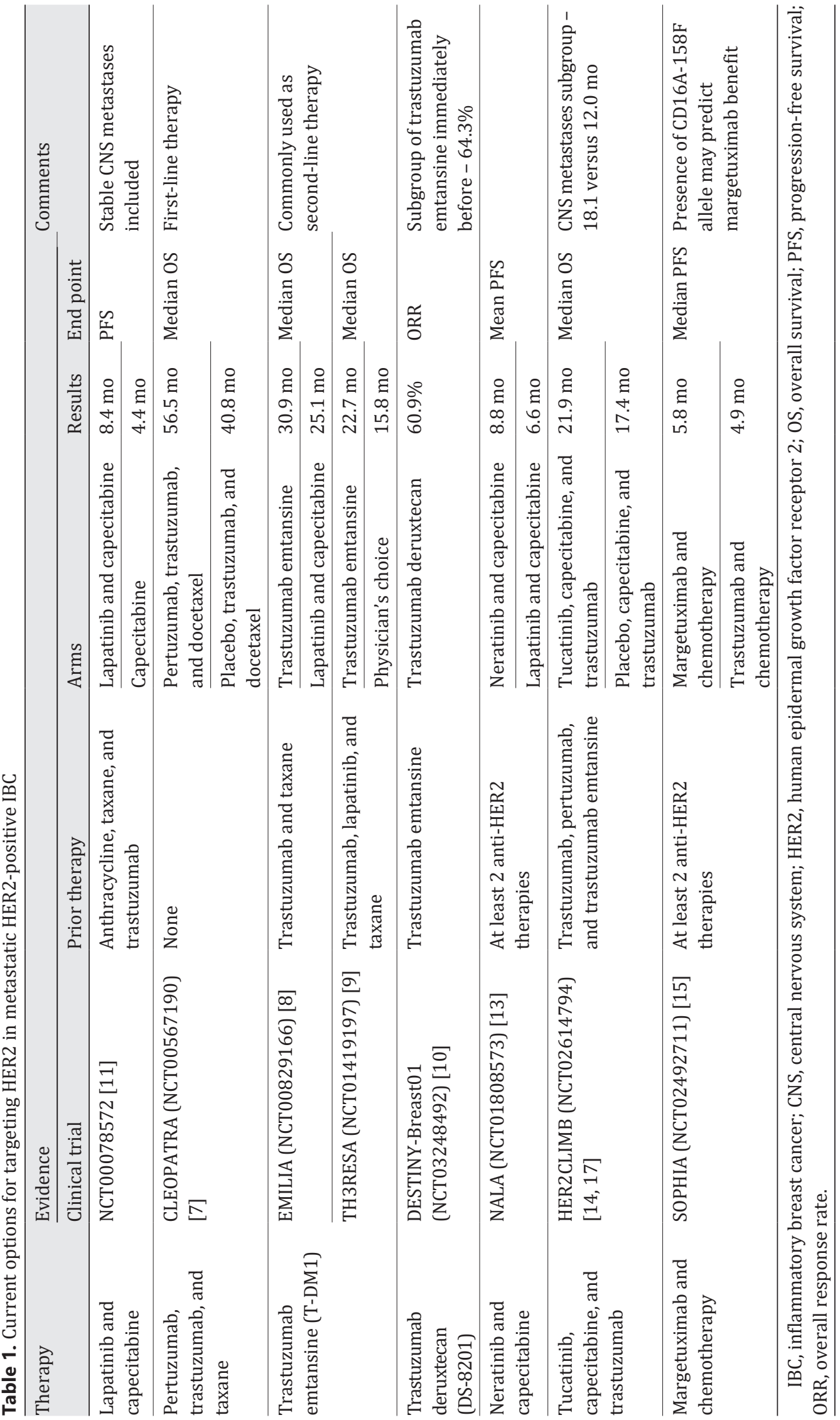


and capecitabine may offer a benefit for IBC patients with CNS disease, although more drugs with better blood-brain barrier penetration are undoubtedly needed.

\section{Conclusion}

A growing number of options for effective systemic therapy are now available for patients with stage IV HER2-positive IBC, which has contributed to a significant prolongation in OS. The success of these drugs attests to the power of developing novel approaches to target HER2. Nevertheless, stage for stage, the prognosis is still worse among patients with IBC compared with non-IBC, regardless of the receptor subtype. Numerous agents are in development, including novel small molecule inhibitors, ADCs, and immunotherapy, which will likely lead to further advancements in the treatment for HER2-positive IBC and continue to translate into prolonged survival among those with de novo metastatic disease. The major challenge will be designing and recruiting for trials specifically for IBC, which will be critical to understanding the unique characteristics of this disease, optimizing treatment strategies, and improving clinical outcomes.

\section{Acknowledgment}

The authors would like to thank Kaitlyn Bifolck for her assistance in manuscript preparation and submission.

\section{Statement of Ethics}

Written informed consent to publish these cases was provided by the patients or their next of kin.

\section{Conflict of Interest Statement}

The authors have no conflicts of interest to declare.

\section{Funding Sources}

This work was supported by the National Cancer Institute F30 CA228208 (J.Y.G.).

\section{Author Contributions}

B.O. conceived and designed the study. J.Y.G. collected the data and drafted the manuscript. J.Y.G. and B.O. reviewed and edited the manuscript. All authors approved the final version of the manuscript.

\section{Karger'k}




\section{Case Reports in Oncology}

\begin{tabular}{l|l}
\hline Case Rep Oncol 2021;14:1071-1079 \\
\hline DOI: 10.1159/000516760 & $\begin{array}{l}\text { ○ 2021 The Author(s). Published by S. Karger AG, Basel } \\
\text { www.karger.com/cro }\end{array}$ \\
\hline
\end{tabular}

Ge and Overmoyer: Prolonged Survival in Metastatic HER2+ Inflammatory Breast Cancer

\section{References}

1 Lim B, Woodward WA, Wang X, Reuben JM, Ueno NT. Inflammatory breast cancer biology: the tumour microenvironment is key. Nat Rev Cancer. 2018;18(8):485-99.

2 Li J, Xia Y, Wu Q, Zhu S, Chen C, Yang W, et al. Outcomes of patients with inflammatory breast cancer by hormone receptor- and HER2-defined molecular subtypes: a population-based study from the SEER program. Oncotarget. 2017;8(30):49370-9.

3 Liu J, Chen K, Jiang W, Mao K, Li S, Kim MJ, et al. Chemotherapy response and survival of inflammatory breast cancer by hormone receptor- and HER2-defined molecular subtypes approximation: an analysis from the National Cancer Database. J Cancer Res Clin Oncol. 2017;143:161-8.

4 van Uden DJP, van Maaren MC, Strobbe LJA, Bult P, van Der Hoeven JJ, Siesling S, et al. Metastatic behavior and overall survival according to breast cancer subtypes in stage IV inflammatory breast cancer. Breast Cancer Res. 2019;21:113.

5 Slamon DJ, Leyland-Jones B, Shak S, Fuchs H, Paton V, Bajamonde A, et al. Use of chemotherapy plus a monoclonal antibody against HER2 for metastatic breast cancer that overexpresses HER2. N Engl J Med. 2001; 344(11):783-92.

6 Gianni L, Eiermann W, Semiglazov V, Lluch A, Tjulandin S, Zambetti M, et al. Neoadjuvant and adjuvant trastuzumab in patients with HER2-positive locally advanced breast cancer (NOAH): follow-up of a randomised controlled superiority trial with a parallel HER2-negative cohort. Lancet Oncol. 2014;15(6):640-7.

7 Swain SM, Baselga J, Kim SB, Ro J, Semiglazov V, Campone M, et al. Pertuzumab, trastuzumab, and docetaxel in HER2-positive metastatic breast cancer. N Engl J Med. 2015;372(8):724-34.

8 Verma S, Miles D, Gianni L, Krop IE, Welslau M, Baselga J, et al. Trastuzumab emtansine for HER2-positive advanced breast cancer. N Engl J Med. 2012;367(19):1783-91.

9 Krop IE, Kim SB, Martin AG, LoRusso PM, Ferrero JM, Badovinac-Crnjevic T, et al. Trastuzumab emtansine versus treatment of physician's choice in patients with previously treated HER2-positive metastatic breast cancer (TH3RESA): final overall survival results from a randomised open-label phase 3 trial. Lancet Oncol. 2017;18(6):743-54.

10 Modi S, Saura C, Yamashita T, Park YH, Kim SB, Tamura K, et al. Trastuzumab deruxtecan in previously treated HER2-positive breast cancer. N Engl J Med. 2020;382(7):610-21.

11 Geyer CE, Forster J, Lindquist D, Chan S, Romieu CG, Pienkowski T, et al. Lapatinib plus capecitabine for HER2positive advanced breast cancer. N Engl J Med. 2006;355(26):2733-43.

12 Kaufman B, Trudeau M, Awada A, Blackwell K, Bachelot T, Salazar V, et al. Lapatinib monotherapy in patients with HER2-overexpressing relapsed or refractory inflammatory breast cancer: final results and survival of the expanded HER2+ cohort in EGF103009, a phase II study. Lancet Oncol. 2009;10(6):581-8.

13 Saura C, Oliveira M, Feng YH, Dai MS, Chen SW, Hurvitz SA, et al. Neratinib plus capecitabine versus lapatinib plus capecitabine in HER2-positive metastatic breast cancer previously treated with $\geq 2$ HER2-directed regimens: phase III NALA trial. J Clin Oncol. 2020;38(27):3138-49.

14 Murthy RK, Loi S, Okines A, Paplomata E, Hamilton E, Hurvitz SA, et al. Tucatinib, trastuzumab, and capecitabine for HER2-positive metastatic breast cancer. N Engl J Med. 2020;382(7):597-609.

15 Rugo HS, Im SA, Cardoso F, Cortés J, Curigliano G, Musolino A, et al. Efficacy of margetuximab vs trastuzumab in patients with pretreated ERBB2-positive advanced breast cancer: a phase 3 randomized clinical trial. JAMA Oncol. 2021;7(4):573-84.

16 Warren LE, Guo H, Regan MM, Nakhlis F, Yeh ED, Jacene HA, et al. Inflammatory breast cancer and development of brain metastases: risk factors and outcomes. Breast Cancer Res Treat. 2015;151:225-32.

17 Lin NU, Borges V, Anders C, Murthy RK, Paplomata E, Hamilton E, et al. Intracranial efficacy and survival with tucatinib plus trastuzumab and capecitabine for previously treated HER2-positive breast cancer with brain metastases in the HER2CLIMB trial. J Clin Oncol. 2020;38(23):2610-9. 